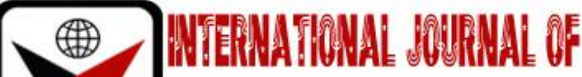

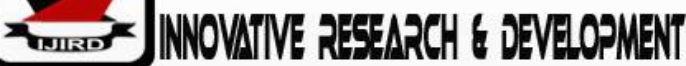

ISSN 2278-0211 (Online)

\section{Effects of the Twelve-Step Facilitation Treatment on Alcohol Consumption among Third Year Students at the University of Cape Coast, Ghana}

\author{
Dr. Christian Evadzi \\ Lecturer, College of Distance Education, University of Cape Coast, Ghana
}

\begin{abstract}
:
Although alcohol consumption among students exists in the university and there are a number of studies to that effect, there is no intervention study that puts the Twelve-Step Treatment to test. The purpose of this study was to ascertain the effects of the Twelve-Step Facilitation Treatment on alcohol consumption among the participants who consume risky and abusive levels of alcohol. Out of 4,653 population, and using Krejcie and Morgan, 160 was selected using simple random technique and administered the questionnaire. Based on this, 51 met the inclusion criteria, however, 40 were selected at random for the intervention. Alcohol Use Disorder Identification Test developed by the WHO was used to collect quantitative data in this Quasi-experimental study. Data were analyzed using One-way ANCOVA where pretest scores were compared to post-test scores after eight weeks of the twelve-step facilitation treatment. The findings showed that the Twelve-Step Facilitation Treatment was effective in reducing alcohol consumption among the participants significantly. It is recommended that University Counsellors should make use of the Twelve-Step Facilitation Treatment in counselling undergraduate students with risky and abusive alcohol consumption behaviour.
\end{abstract}

Keywords: Alcohol, risky consumption, abusive consumption, twelve-step facilitation treatment, group counselling

\section{Introduction}

The use of alcoholic beverages has been an integral part of many cultures for thousands of years (McGovern, 2009). In cultures like that of Egypt and other African countries, alcohol was essential for nutrition, medicine, religious and spiritual ceremonies and it involved inclusion of alcohol beverages in tombs of the dead so that they could be used in afterlife (Chaftetz, 2011). In recent times, alcohol is used in university campuses during birthday and students' representative council week celebrations. Being psychologically dependent on one drink is not a problem but the person who is habituated to getting drunk whenever they feel stressed for instance has a problem. Ghana recorded per capita consumption (15 years and above) as follows: beer - 30\%, wine $-10 \%$, Spirits $-3 \%$, others (locally brewed) $-57 \%$. Per capita consumption of pure alcohol among heavy drinkers stood at 20 litres in 2016 (WHO; cited in Ministry of Health $[\mathrm{MoH}], 2016)$. More so, the protection of the health of populations by preventing and reducing the harmful use of alcohol is a public health priority, and one of the objectives of the World Health Organization is to reduce the health and social burden caused by the harmful use of alcohol (WHO, 1992).

Traditionally, in Ghana, when one is found to be engaged in harmful consumption, some of the remedies resorted to are, family members and friends giving of advice and in some instances, traditional prayers are offered and libation poured. The difficulty however, is that, the efficacy of this approach is not verifiable publicly. There are, however, scientific counselling treatments that are valid and reliable. The Twelve-Step Facilitation Treatment (TSFT) is one of such which is a brief, structured, and manual-driven approach to facilitating early recovery from alcohol abuse, alcoholism, and addiction problems (NREPP, 2008). On one hand, there is evidence that university students engage in risky and abusive alcohol consumption e.g. (Osei-Bonsu, 2017; Oti-Boateng, 2016; Meding, 2012). On the other hand, universities are major hub for talents and skills development and mobilization for national human resource base. Corporate institutions and industries also engage these students upon completion of their programmes as floor, middle and top-level managerial personnel to occupy different roles and make critical decisions. It is, therefore, relevant that, any behaviour among this category of people that is likely to mar their full functioning or lower their productivity in the long run is investigated scientifically.

\section{Literature Review}

\subsection{Tran Theoretical Model (Stages of Change)}

The transtheoretical model (TTM) of behaviour change commonly referred to as the Stages of Change, is a wellknown model in the addictions area developed by Prochaska and DiClemente in the 1970s (LaMorte, 2019). 
The model was originally developed to help people stop smoking. It was determined that, people quit smoking if they were ready to do so. Later, the model was found to be an effective tool in many kinds of change. It is an approach that looks at behaviour change as a process that depends on the person's thinking about alcohol use and problems (Prochaska, Norcross \& DiClemente, 1995). The TTM operates on the assumption that, people do not change behaviour quickly and decisively. Rather, change in behaviour, especially habitual behaviour occurs continuously through a cyclical process. The TTM is not a theory but a model; different behavioural theories and constructs can be applied to various stages of the model where they may be effective (LaMorte, 2019). The TTM posits that individuals move through five stages of change: pre-contemplation, contemplation, preparation, action, maintenance. For each stage of change, different intervention strategies are most effective at moving the person to the next stage of change and subsequently through the model to maintenance which is regarded the ideal stage of behaviour.

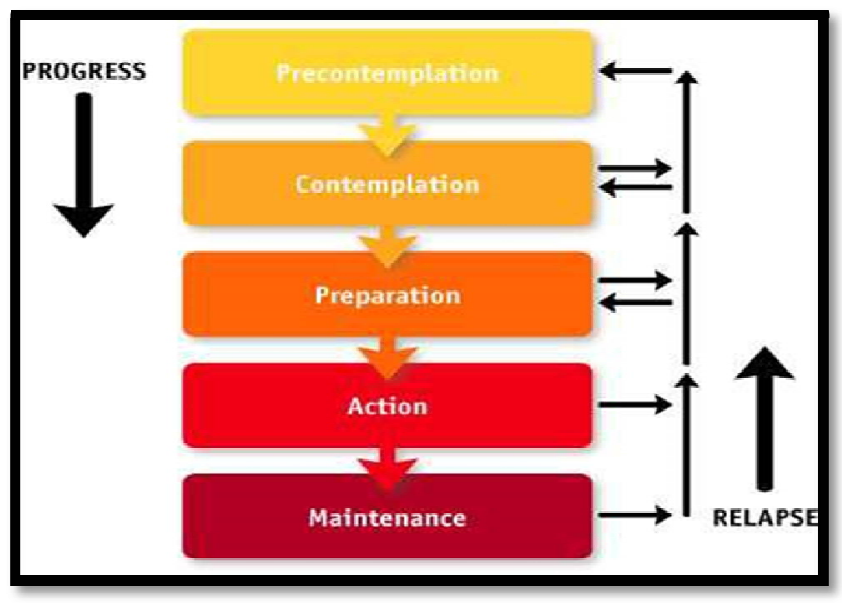

Figure 1: Diagram Illustrating the Stages of Change Source: Prochaska, Norcross \& Diclemente (1995)

\subsubsection{Pre-contemplation}

At this first step, there is some awareness and concern by the victim about drinking excessively or inappropriately, but not real acknowledgment of a problem. (e.g., bosses or family may have said something; the person may have gotten into a fight while drunk, etc.).They may think about changing sometimes, but not in any definite way. At this stage, the Counsellor need to gently help the client come to the conclusion that, they have a problem and there is a reason or a need to change.

\subsubsection{Contemplation}

With this stage, the person decides to do something, although there are probably mixed feelings. The person may be definite that they will change, but not yet.

At this point, the person will be more willing to discuss the pros and cons and options. The counsellor is to help raise awareness of the problem by observation of behaviour.

\subsubsection{Preparation}

An active decision is made and a commitment made to change. The counsellor helps the client gather information, assesses options and considers positive and negative consequences of each option. This is an important stage because this is the beginning of a plan. The plan must be based on enough information and on the client's needs.

\subsubsection{Action}

At this stage, the client is fully committed and starts on the plan using strategies that will lead to achievement of the goal. The counsellor is to reinforce change and provide support and guidance.

\subsubsection{Maintenance}

The person is following the plan and using strategies to stay on track. New habits are practiced. The counsellor is to help the client to support continued change and help with relapse prevention.

Relapse: New habits do not come easily. It needs to be added that, counsellees do not necessarily go through the stages in a linear fashion. Most people will fall back into earlier patterns at least occasionally. It is important to help them understand that they may get off track, but they can plan how to get back on track. Relapses are excellent learning situations for examining what led this to happen and what can be done next time to prevent it from happening again. People may go back and forth between stages several times before a change is complete. Clients should simply be helped to work through the issues and move on again (Prochaska et al., 1995).

\subsection{Studies on Alcohol Consumption}

According to Pew Research Centre (2015), a staggering 90\% of Ghanaians say their religion is very important in their lives. This is relevant to this study because, the TSFT is known for having spiritual inclination where the client seeks 
to entrust their inability to overcome alcohol to a super power. Participants in the study subscribes to Christianity, Islam and African Traditional Believe.

Oti-Boateng (2016) undertook a study titled alcohol consumption among university of Ghana students on Legon campus. It was conducted to determine the proportion of University of Ghana students who consume alcohol and assess the factors and levels of alcohol consumption among University of Ghana students. The study used a cross-sectional design. The dependent variable was alcohol consumption and independent variables were demographic, cultural and social factors. Data were collected using a structured questionnaire and analyzed with STATA version 13. The results showed that, the proportion of students who currently consumed alcohol was $25.81 \%$. More males (33.67\%) consumed alcohol compared to females (17.91\%), and the average age at first consumption of alcohol was 18.67 years. The study further showed that students who smoked were more likely to consume alcohol.

Reavley, Jorm and McCann (2011), studied Alcohol consumption among tertiary education students. The aim of the study was to survey students and staff within a tertiary education institution to investigate patterns of alcohol use, alcohol-related problems. The results showed that, of the completed interviews, 774 (65\%) were students and 422 (35\%) were staff. While staff were more likely to drink regularly, students were more likely to drink heavily. Alcohol consumption was significantly higher in students, in males and in those with a history of earlier onset drinking. In most cases, alcohol-related problems were more likely to occur in students.

It has been reported that university students drink more, on average, than their non-university peers (Schulenberg, Maggs, Long, Sher, Gotham, Baer, Kivlahan, Marlatt, \&Zucker, 2001). Also, in a study conducted by Wechsler, Lee, Kuo, Seibring, Nelson and Lee (2002), heavy drinking rates had remained stable, while extreme drinking (including drinking to get drunk and frequent drunkenness) had significantly increased over the past ten years (Wechsler et. al., 2002).

University campuses, then, may be places that nurture drinking, as opposed to repelling it. Pagano et al., (2004) found that providing support to others may benefit recovering individuals because it increases a commitment to abstinence, satisfaction from helping other individuals in need, and the helper's own sense of independence and selfefficacy. In fact, recovering individuals who help their peers to maintain long-term sobriety are better able to maintain sobriety themselves. The facilitation of the TSFT allows for peer help. Patients who engage in more helping during treatment tend to be more involved after treatment and, in turn, are more likely to achieve abstinence. Moreover, clients who become sponsors are more likely to maintain abstinence than (Zemore et al. 2004).

School-based brief intervention studies suggest that reductions in alcohol use and consequences are mediated by purposeful self-change efforts on the part of teens (Brown, Anderson, Ramo, Tomlinson, 2005) and that expectations of reduction or cessation outcomes may be critical to this change process (Metrik et al. 2004). One 4-year follow up of college freshmen found that reduction in consequences had a lasting effect, whereas reductions in quantity and frequency of alcohol use had washed out by then (Baer, Kivlahan, Blume, 2001).

According to Kelly et al. (2002), attendance at 12-step meetings in the first 3 months after treatment was associated with more motivation for abstinence and self-efficacy at 3-month follow-up, which predicted abstinence at 6month follow-up. The strength of affiliation with SHGs explained part of the connection between 12-step attendance and motivation for abstinence, which explained some of the link between attendance and 6-month outcomes.

Patients with both Substance Use Disorders (SUDs) and posttraumatic stress disorder whose identity matched twelve-step philosophy participated more in Self Help Group (SHG) activities; more participation was associated with less distress for these patients but with more distress for patients who did not have a 12-step identity (Ouimette et al., 2001).

Affiliation with twelve-step also tends to promote more reliance on behaviorally oriented substance use coping processes. In this respect, Snow et al. (1994) found that individuals who were more involved in Alcohol Anonymous were more likely to rely on specific coping responses aimed toward reducing substance use, such as spending time with nondrinking friends, talking to someone about their drinking problems, rewarding themselves for trying to stop drinking, and becoming more aware of social efforts to help people stop drinking.

\section{Methodology}

Quasi-experimental design using quantitative approach was used to ascertain the effectiveness of the Twelve-Step Facilitation Treatment. Quantitative research is an approach for testing objective theories by examining the relationship among variables. These variables, in turn, can be measured, typically on instruments, so that numbered data can be analyzed using statistical procedures (Creswell, 2014). The WHO's Alcohol Use Disorder Identification Test (AUDIT) questionnaire was adapted for the study. The 10-item instrument includes questions to assess alcohol intake (questions 13), alcohol dependence (questions 4-6) and alcohol related problems (questions 7-10). The questionnaire was pilot tested among 40 University of Ghana third year students and its reliability was confirmed with Cronbach's alpha level of .80.

This intervention study through group counselling treatment was carried out within a period of 8weeks during the academic year of 2018-2019. 40, being third year undergraduate students were involved in the study with age ranges of (17-20), (21-24) and (25+) and gender distribution of 28 males and 12 females. The third years were considered for the study because of assimilation. The fourth (final) year students were most ideal, however, they were not considered because they were exiting and might not be available for the treatments. Third year students were therefore considered. They have spent more years in the university campus than the first and the second year and were more likely to be engrossed in the phenomenon. Out of 4,653 population, and using Krejcie and Morgan, 160 was selected using simple random technique and administered the questionnaire. Based on this, 51 met the inclusion criteria, however, 40 was selected at random for the intervention. 
The 40 were put into two groups of 20 per group. Group 1 as the experimental group (13 - males, 7 - females) and group two as the control group (15 - males, 5 - females). The determination of the number per group was influenced by Creswell (2012) who asserts that, approximately 15 participants in each group is desirable for experimental study in education. Again, a number of researchers have indicated that, the number for group counselling can range from 15 to 20 (Kagu, 2010; 2017; Ohanaka \& Ofuani, 2018). Similarly, Adzaku, Awabil and Forde (2017), in an intervention study conducted in Ghana also used 20 participants per group. One-way ANCOVA was used to analyze the data collected on the hypothesis. It was appropriate to use because the hypothesis was seeking to determine effects while controlling for their pre-test scores in experimental sense i.e. control and experimental groups. More specifically, the hypothesis had only one independent variable i.e. groups (twelve-step, and control) hence the use of the one-way ANCOVA.

\section{Findings}

$\mathrm{H}_{\mathrm{A}} 1$ : There is significant effect of the twelve-step facilitation treatment on the alcohol consumption among participants. This hypothesis was geared towards examining the effect of twelve-step facilitation treatment on the alcohol consumption of students. In order to determine the effects of this treatment, the post-test scores of the treatment group was compared with the control group, while controlling for their pre-test scores. To perform this analysis, one-way ANCOVA test was used. Table 1 presents the results.

\begin{tabular}{|c|c|c|c|c|c|}
\hline & & Mean & & & Partial Eta \\
\hline Source & $\mathrm{df}$ & Square & $F$ & Sig. & Squared $\left(\eta_{p}{ }^{2}\right)$ \\
\hline Corrected Model & 2 & 358.53 & 83.17 & $<.001$ & .817 \\
\hline Intercept & 1 & 110.61 & 25.66 & $<.001$ & .314 \\
\hline Pre-test & 1 & 1.35 & .31 & .579 & .006 \\
\hline Group & 1 & 535.48 & 124.22 & $<$ & $.001^{*}$ \\
\hline Error & 36 & 4.31 & & & \\
\hline Total & 40 & & & & \\
\hline Corrected Total & 39 & & & & \\
\hline
\end{tabular}

Table 1: ANCOVA of Tests of Between-Subjects Effects Comparing Post-test Scores of Groups

A one-way ANCOVA test was performed to compare the post-test scores of control and twelve-step facilitation treatment group on the alcohol consumption while controlling for their pre-test scores. The independent variable was the groups, which had two levels: control and twelve-step facilitation treatment. The dependent variable was the post-test scores, which was measured on interval scale, and the covariate was the pre-test scores which were also measured on interval scale.

The results in Table 1 indicate a statistically significant difference in the post-test scores of the participants in the two groups after controlling for their pre-test scores, $F(1,36)=124.22, p<.001, \eta p^{2}=.82$. The magnitude of the difference was large, and this implies that $82 \%$ of the variance in post-test scores (alcohol consumption) was explained by the groups. The results are presented in Table 2.

\begin{tabular}{|c|c|c|}
\hline Group & Mean & Std. Error \\
\hline Control & $15.33^{\mathrm{a}}$ & .47 \\
\hline 12-step & $5.07^{\mathrm{a}}$ & .48 \\
\hline
\end{tabular}

Table 2: Adjusted Means on Post-Test

a. Covariates Appearing in the Model Are Evaluated at the Following Values: Pre-Test $=16.10$

From Table 2, after controlling for the pre-test scores, the post-test scores for the control group $(M=15.33, S D=$ $.47)$, was higher than the twelve-step facilitation treatment group $(M=5.07, S D=.48)$. The implication of this is that, the twelve-step facilitation treatment was effective in reducing the alcohol consumption level of the students. In effect, twelve-step facilitation treatment was effective in reducing alcohol consumption of students. Based on the results of this study, the null Hypothesis was rejected in favour of the alternative hypothesis.

5. Discussions

The study revealed that, the twelve-step facilitation treatment was effective in reducing the alcohol consumption level among the students significantly. This result supports the findings revealed by Kelly et al. (2002) that attendance at twelve-step meetings was associated with more motivation for abstinence and self-efficacy. This implies that the twelvestep facilitation counselling treatment did record positive effects on participants' alcohol consumption behaviour. 
Therefore, the treatment is effective in reducing risky and abusive alcohol consumption behaviour among the students. Again, the current result is in line with the findings obtained by NIAAA (2000) that, brief interventions are especially effective as a preventive measure that helps people make changes in the early stages of risky drinking.

Perhaps, the participants responded well to the treatment because of the nature and procedures of the treatment coupled with the dexterity with which the facilitator carried out the facilitation where the counsellees were involved and engaged meaningfully. There are assumptions, approaches and skills involved in using the twelve-step facilitation which when followed, can yield a positive result. For instance, the participants were engaged in a meaningful and a productive way observing all the protocols and quality engagements which could be a factor in the result arrived at. This assertion is supported by Snow et al. (1994) who found that, individuals who were more involved in AA meetings were more likely to rely on specific coping responses aimed toward reducing substance use such as spending time with non-drinking friends, talking to someone about their drinking problems, rewarding themselves for trying to stop drinking and becoming more aware of social efforts to help people stop.

Also, the twelve-step facilitation is group-oriented which allow the counsellees to experience social support from other counsellees in treatment. The ideology of the twelve-step again expects individuals with substance use problems to admit past wrongdoing, acknowledge inability to control substance use, and trust a higher power to achieve recovery. This might have also contributed to the twelve-step facilitation treatment being effective in reducing risky and abusive alcohol consumption behaviour. To support this argument, Pagano et al. (2004) stated that, recovering individuals who help their peers to maintain long-term sobriety are better able to maintain sobriety themselves. Zemore et al. (2004) also indicated that, clients or counsellees who engage more in helping during treatment tend to be more involved in Self Help Groups (SHGs) after treatment and, in turn, are more likely to achieve abstinence. Each step of the twelve-step facilitation treatment involves sharing personal experiences, probing other participants' experiences and the counselees helping others to understand their predicaments and also helping themselves and others. In effect, the group tends to offer guidance, goal direction and monitoring through the modelling of substance use refusal skills and all of these could enhance the social support gained from the twelve-step thereby making it efficacious.

It is also an established fact that, with the twelve-step facilitation, individuals whose beliefs are in consonant with the twelve-step orientation i.e. spiritual inclination and a belief in a super power are more likely to affiliate with the twelve-step facilitation treatment hence benefit from it. An evidence was established when Ouimette et al. (2001) found out that, counsellees with substance use disorders whose identity matched the twelve-step philosophy participated more in self-help group activities; more participation was associated with less distress for these counsellees but with more distress for counsellees who did not have a twelve-step identity.

According to Pew Research Centre (2015), a staggering 90\% of Ghanaians say their religion is very important in their lives. For this current study, all of the participants were affiliated spiritually. They all showed a belief in a super power (God) by subscribing to Christianity, Islam and African Traditional Belief. It therefore comes to reason that, the twelve-step facilitation treatment used was in consonance with the participants orientation spiritually and therefore contributed to the effectiveness.

\section{Conclusions}

Based on the results from the main data, the following conclusions were arrived at: The study found out that the counselling treatment (the twelve-step facilitation) used for the intervention was effective in reducing alcohol consumption among the participants significantly. The study, therefore, concludes that, in counselling situations, involving risky and abusive alcohol consumption among undergraduate university students, the twelve-step facilitation treatment can reduce the level of consumption significantly. In other words, the twelve-step facilitation treatment is effective to use.

\section{References}

i. Adzaku, S. D. Y., Awabil, G., \& Forde, L. D. (2017). Improving the study behaviour of Ghanaian senior high school students through counselling. Ghana Journal of Education, 3, 1-14.

ii. Baer, J. S., Kivlahan, D. R., \& Blume, A. W. (2001). Brief intervention for heavy-drinking college students: 4year follow-up and natural history. American Journal of Public Health, 91, 1310-1316.

iii. Brown, S. A., Anderson, K. G., Ramo, D. E., \& Tomlinson, K. L. (2005). Treatment of adolescent alcoholrelated problems: A translational perspective. In M. Galanter, (Ed.), Recent developments in alcoholism, Vol. 17: Alcohol problems in adolescents and young adults: Epidemiology, neurobiology, prevention, treatment (pp.327-348). New York, NY: Springer.

iv. Chafetz, M. (2011). The evolution of alcohol in society. Retrieved fromhttp://blocs.wbc.co/theevolution.of.alcohol.in society.

v. Creswell, J. W. (2014). Research design: Qualitative, quantitative and methods approaches (4th ed.). California, CA: Sage Publication.

vi. Creswell, J.W. (2012). Educational research: Planning, conducting and evaluating quantitative and qualitative research (4th ed.). Boston, MA: Tex Tech Int.

vii. Kagu, B. (2010). Influence of group counselling on learning and remembering strategies of diploma students at the University of Maiduguri. International Journal of Guidance and Counselling, 23(1),137-143.

viii. Kelly, J. F., Myers, M. G., \& Brown, S.A. (2002). Do adolescents affiliate with 12-step groups? A multivariate process model of effects. Journal of Studies on Alcohol, 63, 293-304.

ix. LaMorte, W. W. (2019). The transtheoretical model: Stages of change. Retrieved from http://sphweb.bumc.bu.edu/otlt/MPH- Modules/SB/Behavioral Change Theories. 
x. Meding, B. (2012). Interactions between attendance and students' alcohol consumption (Unpublished Master's thesis). Retrieved from http://thekeep.eiu. edu/theses/784.

xi. McGovern, P. (2009). Uncorking the past: The quest for wine, beer, and other alcoholic beverage. Berkley, CA: University of California Press.

xii. class

xiii. Metrik, J., Gunn, R. L., Jackson, K. M., Alexander, W. Sokolovsky, A. W., \&Borsari, B. (2018). Daily patterns of marijuana and alcohol-use among individuals with alcohol and cannabis use disorders. Alcohol ClinExp Res., 42(6), 1096-1104.

xiv. Ministry of Health. (2016). Ghana national alcohol policy. Accra: Augitive.

xv. National Institute on Alcohol Abuse and Alcoholism. (NIAAA). (2000). Alcohol involvement over the life course. In: Tenth special report to the U.S. congress on alcohol and health: Highlights from current research (pp. 28-53). Bethesda, MD: Dept. of Health and Human Services, NIAAA.

xvi. NREPP. (2008b). Twelve-step facilitation therapy. Retrieved http://www.nrepp.samhsa.gov/programfulldetails.asp.

xvii. Ohanaka, B. I., \& Ofuani, F. N. (2018). Age and gender differences in enhancing senior secondary school students study habits through reading and group counselling. Creative Education, 117(3), 45-56.

xviii. Osei-Bonsu, E. (2017). Prevalence of alcohol consumption and factors influencing alcohol use among the youth in Tokorni-Hohoe, Volta Region of Ghana. Science Journal of Public Health, 5, 205-206.

xix. Oti-Boateng, N. Y. (2016). Alcohol consumption among university of Ghana students on Legon campus (Unpublished Master's thesis). University of Ghana, Legon.

Xx. $\quad$ Ouimette, P. C., Humphreys, K.., \& Moos, R. (2001). Self-help participation and functioning among substance use disorder patients with posttraumatic stress disorder. J Subst Abuse Treat, 20, 25-32.

xxi. Pagano, M. E., Friend, K. B., \& Tonigan, J. S. (2004). Helping other alcoholics in Alcoholics Anonymous and drinking outcomes: $\quad$ Findings from Project MATCH. J Stud Alcohol 65, 766-773.

xxii. Pew Research Centre. (2015). Global attitudes survey. Washington, DC: PRC.

xxiii. Prochaska, J. O., Norcross, J. C., \& DiClemente, C. C. (1995). Changing for good. New York, NY: Avon Books.

xxiv. Reavley, N. J., Jorm, A. F., \& McCann, T. V. (2011). Alcohol consumption in tertiary education students. BMC Public Health 11, 545-546.

xxv. Schulenberg, J., Maggs, J. L., Long, S.W., Sher, K. J., Gotham, H. J., $\quad$ Baer, J. S., Kivlahan, D. R., Marlatt, G.A., \& Zucker, R. A., (2001). The problem of college drinking: Insights from a developmental perspective. Alcoholism: Clinical and Experimental Research, 25(3), 473-477.

xxvi. Snow, M. G., Prochaska, J. O., \& Rossi, J. S. (1994). Processes of change in Alcoholics Anonymous: Maintenance factors in long- term sobriety. J Stud Alcohol, 55, 362-371.

xxvii. Wechsler, H., Lee, J. E., Kuo, M., Seibring, M., Nelson, T. F., \& Lee, H. (2002). Trends in college binge drinking during a period of increased prevention efforts. Journal of American College Health, 50, 203-217.

xxviii. World Health Organization. (1992). The ICD-10 Classification of mental and behavioural disorders diagnostic criteria for research. Geneva, Switzerland: World Health Organization.

xxix. Zemore, S. E., Kaskutas, L. A., \& Ammon, L. N. (2004). In 12-step groups, helping helps the helper. Addiction, 99, 1015-1023.

\section{Appendix}

Alcohol Consumption Inventory

Questionnaire (Adapted From Audit)

University of Cape Coast

Department Of Guidance and Counselling

\section{ACI Questionnaire for Students}

This seeks to ascertain the level of alcohol consumption among students. In view of this, I request of you to kindly fill this questionnaire. Note that your response to the questions will be used for academic purpose and will be treated confidential.

\section{Section A: Background Information}

1. Gender: Male [] Female []

2. Age: 17 - 20yrs [ ] $21-24 y r s[] \quad 25 y r s$ and above []

3. Religion: Islam [ ] Christianity [] African Tradition [] Others []

Section B. Alcohol Consumption Inventory

Kindly answer the following by ticking $(\sqrt{ })$ inside the appropriate column for each question 


\begin{tabular}{|c|c|c|c|c|c|c|}
\hline $\mathbf{S} / \mathbf{N}$ & Question Item & & & Scores & & \\
\hline & & $\mathbf{0}$ & 1 & 2 & 3 & 4 \\
\hline \multirow[t]{3}{*}{4} & How often do you & Never & Monthly & 2 to 4 & 2 to 3 & 4 or more \\
\hline & have a drink & & or less & times a & times a & times a \\
\hline & containing alcohol? & & & Month & week & Week \\
\hline \multirow[t]{5}{*}{5} & How many drinks & None & 2 to 4 & 5 or 6 & 7 to 9 & 10 or \\
\hline & containing alcohol do & & & & & More \\
\hline & you have on a typical & & & & & \\
\hline & day when you are & & & & & \\
\hline & drinking? & & & & & \\
\hline \multirow[t]{5}{*}{6} & How often do you & Never & Less than & Monthly & Weekly & Daily or \\
\hline & have more than five & & monthly & & & Almost \\
\hline & or more alcoholic & & & & & Daily \\
\hline & drinks on one & & & & & \\
\hline & occasion? & & & & & \\
\hline
\end{tabular}

Table 3: Alcohol Consumption Inventory 